\title{
Oclusiones Crónicas
}

\author{
Humberto Torres
}

Las oclusiones coronarias crónicas (OCC), definidas como obstrucción completa de una arteria coronaria con flujo TIMI 0 por más de 3 meses de evolución ${ }^{1}$, tienen una incidencia de un 30 a $50 \%$ dentro de las lesiones encontradas en los pacientes con cardiopatía coronaria y aumenta más en los pacientes mayores ${ }^{2}$. Sin embargo constituyen sólo entre un 10-15\% de todas las angioplastías coronarias percutáneas (ACP) ${ }^{3}$. La baja incidencia en el abordaje de este tipo de lesiones, estaba determinada por el alto número de fracasos en el intento de atravesar la obstrucción con el alambre guía como también por el alto porcentaje de reestenosis ${ }^{4-5}$. Esto inclinaba la terapia a la revascularización quirúrgica o al tratamiento médico. En los últimos años ha mejorado la experiencia en este tipo de lesiones, desarrollándose nuevas estrategias, como el uso de guías paralelas, la inyección simultánea bilateral, para evaluar el correcto avance de la guía, el uso de IVUS y el abordaje retrógrado. Además, se han incorporado guías coronarias de mayor rendimiento y stents medicados con menos \% de reestenosis, lo que ha permitido mejorar el rendimiento de la $\mathrm{ACP}$ en las $\mathrm{OCC}$, renovando el interés en su abordaje con un éxito actual de alrededor de un $80 \% .^{6-7}$.

Por otro lado existen evidencias que el manejo exitoso de las OCC es determinante en el control sintomático del paciente coronario, la mejoría de la función ventricular y en la sobrevida a largo plazo, siempre que esté confirmado el rol isquémico del vaso ocluido ${ }^{3-8}$. Esto es fundamental, puesto que estas intervenciones no están exentas de riesgo, de allí que deben estar justificadas por la demostración de isquemia y/o viabilidad del vaso a tratar.

En estos avances ha sido primordial el aporte de grupos japoneses que han sido pioneros en muchas de las técnicas anteriormente descritas y que se han expandido a todo el mundo. ${ }^{7}$

En nuestro país han aparecido experiencias en este tipo de lesiones, como ésta del grupo del Hospital Barros Luco Trudeau de Santiago, en que nos presenta una serie importante de 90 pacientes con OCC tratados con ACP, sus resultados, la confección de un score para caracterizar la complejidad de las OCC y también el análisis del rendimiento de distintos materiales de acuerdo a la complejidad de la lesión tratada ${ }^{9}$. Del análisis de esta serie destacan dos aspectos: primero la confección de un score para identificar la complejidad de las obstrucciones y segundo la evaluación de distintas guías y balones utilizados en el procedimiento de estos pacientes. Con respecto al primer punto, me parece de interés la confección de un score local que permita estratificar las lesiones de acuerdo a su complejidad, porque de ésta manera se puede planificar mejor el procedimiento, dejando los casos más difíciles para la primera hora del día, ahorrar contraste y radiación al máximo, usar abordaje bilateral, considerar acceso retrogrado, etc. Además, se pueden evaluar mejor los resultados en poblaciones comparables y también la mejoría de los resultados en el tiempo. La literatura demuestra que los factores determinantes de complejidad han ido variando con el tiempo, en la medida que los grupos adquieren experiencia, por esto es importante evaluar periódicamente los factores analizados porque, sin duda, se acercaran a lo que se publica en las series actuales, donde el largo del segmento obstruido, las tortuosidades del vaso afectado y la presencia de calcificación son las características más relevantes de complejidad ${ }^{7}$. El carácter retrospectivo de esta muestra tiene las limitantes de sesgo por selección de los casos, por la experiencia variada del operador principal y de una estrategia no uniforme en el abordaje de estos casos, pero de todas maneras es un esfuerzo interesante para el desarrollo del abordaje de las OCC en nuestro país. El segundo aspecto, que es la elección de las guías y balones me parece más discutible ya que se usó una gran diversidad de guías de diferentes 
características, algunas con una frecuencia muy baja de uso, dentro de ellas, las guías dedicadas a OCC y que se eligieron de acuerdo a la experiencia del operador y seguramente dependiendo también de la disponibilidad de material, por lo que me parece que no hay un panorama claro de qué insumo utilizar. Por otra parte, se sabe que en las OCC con microcanales, lo ideal es utilizar guías hidrofílicas idealmente con punta más pequeña para penetrar estos canales, o en los casos de obstrucción por reestenosis intrastent también las guías hidrofílicas van a tener un excelente rendimiento. En las lesiones sin comunicación el acercamiento a la lesión debe hacerse con una guía floppy montada en un balón sobre la guía o un microcatéter, una vez en la zona de oclusión cambiar por una guía de perforación (driling) de peso progresivo hasta llegar a las guías de penetración cuando no se consigue atravesar el segmento ocluído. Si hay mucho calcio probablemente vamos a pasar más rápidamente a las guía de penetración puesto que la dureza de la obstrucción hace poco probable que una guía hidrofílica o de perforación pue- dan tener éxito. Por esto más que una elección aleatoria de experiencia individual, ya tenemos un conocimiento previo que nos permite elegir de un armamentario un poco más acotado y así disminuir los tiempos de estos procedimientos y mejorar las posibilidades de éxito.

Por otro lado, en relación a los balones, que en general no es el mayor problema, se elegirán los que tengan el mejor perfil de cruce, por lo que la elección se reduce a los balones más pequeños de diferentes marcas y características.

Sin duda que los avances en experiencia y en material hacen más asequibles las OCC al tratamiento percutáneo con buenas chances de éxito a corto y largo plazo con el consiguiente impacto en control de síntomas, mejoría en función ventricular y de mortalidad. Por esto es que a pesar de lo complejo, estas lesiones serán abordadas cada vez más en las series de ACP de nuestro país. El reto pendiente es mejorar los resultados en las lesiones complejas y, en ese sentido, esta experiencia del Dr. Pedreros y col. ${ }^{9}$ es un excelente aporte para ello.

\section{Referencias:}

1. Stone GW, Kandzari DE, Mehran R, Colombo A. Percutaneous recanalization of chronically occluded coronary arteries. Circulation 2005; 112: 2364-2372.

2. Christofferson RD, Lehmann KG, Martin GV, Every N, Caldwell $\mathrm{JH}$, Kapadia SR. Effect of chronic total occlusion on treatment strategy. Am J Cardiol 2005; 95: 1088-1091.

3. Hoye A, Van Domburgh RT, Sonnenschein K, Serruys PW. Percutaneous coronary intervention for chronic total occlusions: a Thoraxcentre experience 1993-2002. Eur Heart J 2005; 36: 26302636.

4. Stone GW, Rutherford BD, McConahay DR, Johnson WL Jr, Giorgi LV, Ligon RW, et al. Procedural outcome of angioplasty for total coronary artery occlusion: an analysis of 971 lesions in 905 patients. J Am Coll Cardiol 1990; 15: 849-856.

5. Bell MR, Berger PB, Bresnahan JF, Reeder GS, Bailey KR, Holmes DR Jr.. Initial and long term outcome of 354 patients after coronary balloon angioplasty of total coronary artery occlusions. Circulation 1992; 85: 1003-1011.
6. Suttorp MJ,Laarman GJ, Rahel BM, Kelder JC, Bosschaert MA, Kiemeneij F, et al. Primary stenting of totally occluded native coronary arteries. (PRISON II): A randomized comparison of bare metal stent implantation with sirolimus eluting stent implantation for the treatment of total coronary occlusions. Circulation 2006; 114: 921-928.

7. Rathore S, Matsuo H, Terashima M, Kimura M, Tsuchikane E, Katoh O, Suzuki T, et al. Procedural and in hospital outcomes after percutaneous coronary intervention for chronic total occlusions of coronary arteries 2002 to 2008. J Am Coll Cardiol Interv; 2009; 6: 489-497.

8. Suero JA, Marso SP, Jones PG, Laster SB, Huber KC, Giorgi LV, et al. Procedural outcomes and long trerm survival among patients undergoing percutaneous coronary intervention of a chronic total occlusion in native coronary arteries: a 20 years experience. J Am Coll Cardiol 2001; 38: 409 - 414.

9. Pedreros P, Lamich R, Aguirre A, Romero C, Chamorro H. Intervencionismo en oclusiones crónicas, evaluación de la efectividad de cruce de distintas guías y balones según complejidad. Rev Chil Cardiol 2010; 29: 299 - 305. 